\title{
Nationwide survey to evaluate medical utilization by patients with inguinal hernia and the risk of developing varicocele in Taiwan
}

This article was published in the following Dove Press journal:

Patient Preference and Adherence

20 January 2014

Number of times this article has been viewed

\author{
Wen-Liang Liu',** \\ Yu-An Chen ${ }^{2}$ \\ Yu-Wei Lai ${ }^{3,4, *}$ \\ Thomas $Y$ Hsueh $^{3,4}$ \\ Shiou-Sheng Chen ${ }^{3,4}$ \\ Allen W Chiu ${ }^{4}$ \\ 'Taipei Databank for Public Health \\ Analysis, Institute of Clinical Research \\ and Teaching, Taipei City Hospital, \\ ${ }^{2}$ Institute of Preventive Medicine, \\ National Taiwan University, ${ }^{3}$ Division \\ of Urology, Taipei City Hospital Renai \\ Branch, ${ }^{4}$ Department of Urology, \\ School of Medicine and Shu-Tien \\ Urological Institute, National Yang- \\ Ming University, Taipei, Taiwan
}

"These authors are joint first authors

Correspondence: Shiou-Sheng Chen Division of Urology, Taipei City Hospital Renai Branch, I0 Renai Street, Section 4, Taipei 106, Taiwan Tel +88 6227093600 ext 363 I

Fax +88 6227045064

Email eric.yoyo@msa.hinet.net
Background: The purpose of this study was to analyze age, geographical and seasonal variations in medical service utilization by patients with inguinal hernia in Taiwan, and the influence of herniorrhaphy on development of ipsilateral varicocele in male patients.

Methods: Between 2001 and 2008, comprehensive data on the characteristics of medical service utilization by patients with inguinal hernia was evaluated via a retrospective nationwide population-based study. Data were obtained from the National Health Insurance Research Database in Taiwan. Parameters for comparison included newly diagnosed inguinal hernia cases, number of herniorrhaphies, and incidence rates every year, number of outpatient visits for inguinal hernia, and herniorrhaphy by age, season, and area of Taiwan.

Results: There was an average of 1,466 newly diagnosed inguinal hernia cases and 871.9 herniorrhaphies performed per year per million population during the study period. The male ratio for both newly diagnosed inguinal hernia cases and number of herniorrhaphies increased significantly by age. The number of newly diagnosed inguinal hernia cases and outpatient visits for inguinal hernia was highest during summer, followed by spring, autumn, and winter, and in the north of Taiwan, followed by the center, south, and east. Additionally, the incidence of developing ipsilateral varicocele after herniorrhaphy was low in male patients.

Conclusion: The number of newly diagnosed inguinal hernia cases and outpatient visits for inguinal hernia is highest during summer and lowest in eastern Taiwan. In addition, the incidence of developing ipsilateral varicocele after herniorrhaphy is higher in patients aged 10-19 years.

Keywords: age, herniorrhaphy, inguinal hernia, season, varicocele

\section{Introduction}

Inguinal hernia is a common disease in males, with an incidence of $1 \%-2 \% .{ }^{1}$ In the US, inguinal hernia is more common in children and elderly males, while heavier men may have a lower risk. ${ }^{2}$ Inguinal hernia is one of the most common medical problems requiring an operation; however, the etiology remains uncertain. ${ }^{3}$ Some studies have suggested that an inguinal hernia can be caused by increased intra-abdominal pressure and that some varicocele patients may suffer from scrotal pain after strenuous exercise. ${ }^{4}$ An open processus vaginalis and strenuous exercises are widely accepted as risk factors for inguinal hernia; ${ }^{5}$ however, many others have also been reported, including genetic predisposition, sex, and age, as well as variations in collagen amount, type, structure, and degradation rate. ${ }^{5-10}$ Ribarski et al reported that mutation $1090 \mathrm{C}>\mathrm{T}$ in ubiquitinspecific protease 26 on $\mathrm{X}$ chromosomes may be a genetic risk factor for developing inguinal hernia, and that this may be associated with male infertility. ${ }^{11}$ Further, about $69 \%-81 \%$ of men with secondary infertility have a varicocele. ${ }^{12,13}$ Coincidental findings 
of varicocele and inguinal hernia are expected to become more frequent considering the pathogenesis of these disorders; however the correlation between the two is unclear.

Information concerning utilization of medical services by patients with inguinal hernia in terms of age, season, and area is relatively scarce. This nationwide study aimed to evaluate utilization of medical services by patients with inguinal hernia via calculation of newly diagnosed inguinal hernia cases, number of herniorrhaphies performed each year, number of outpatient visits for inguinal hernia, and number of herniorrhaphies by age, season, and area of Taiwan. In addition, we evaluated the influence of herniorrhaphy on developing ipsilateral varicocele in male patients. Data were obtained from the National Health Insurance Research Database in Taiwan from 2001 to 2008 .

\section{Materials and methods}

Since its establishment in 1995, the National Health Insurance program has covered approximately $96 \%$ of the population, $97 \%$ of hospitals, and $90 \%$ of clinics in Taiwan. ${ }^{14,15}$ To ensure the accuracy of the claims data, the Bureau of National Health Insurance performs expert reviews on a random sample of every 50-100 claims in each hospital and clinic quarterly, and imposes a severe penalty on health providers if they violate the regulations. All data from the National Health Insurance Research Database were deidentified. Comprehensive data for characteristics of medical service utilization were evaluated using parameters that included newly diagnosed inguinal hernia cases, incidence of newly diagnosed inguinal hernia cases each year, number of herniorrhaphies, number of outpatient visits for inguinal hernia, and number of herniorrhaphies by age, season, and area of Taiwan. Patient age was determined by date of birth, while geographical area was documented either by residential area or location of employment. ${ }^{16}$ Meteorological data were obtained from the Central Weather Bureau in Taiwan. ${ }^{17}$

The diagnosis of inguinal hernia was according to International Statistical Classification of Disease and Related Health Problems, 9th edition (ICD-9-CM) codes, 550.00-550.03, 550.10-550.13, and 550.90-550.93 for inguinal hernia and 456.4 for varicocele, and surgical National Health Insurance classification codes (75606B, 75607C, 75613C, and 75614C) for herniorrhaphy. This study was exempted from survey by the institutional review board of Taipei City Hospital.

\section{Statistical analysis}

We used $\chi^{2}$ tests to analyze the experimental data. Statistical Package for the Social Sciences version 17.0 software for
Windows (SPSS Inc, Chicago, IL, USA) was used for all statistical analyses. A $P$-value $<0.05$ was considered to be statistically significant.

\section{Results}

Data on newly diagnosed inguinal hernia cases and number of herniorrhaphies between 2001 and 2008 were stratified by year (Table 1) and by age (Table 2). The number of newly diagnosed inguinal hernia cases, number of outpatient visits for inguinal hernia, and cases that received herniorrhaphy were stratified by season (Table 3), and the number of newly diagnosed inguinal hernia cases and outpatient visits for inguinal hernia were stratified by area of Taiwan (Table 4). Temperature was highest in the summer (June, July, and August), followed by spring (March, April, and May), autumn (September, October, and November) and winter (December, January, and February). Numbers of newly diagnosed varicocele cases after herniorrhaphy were stratified by year and age (Table 5).

From 2001 to 2008, there were an average of 1,466 newly diagnosed inguinal hernia cases per year per million population and 871.9 herniorrhaphies per year per million population. The overall incidence of newly diagnosed inguinal hernia cases was 1.52 (per $10^{3} /$ year) and the overall incidence of herniorrhaphy was 0.86 (per $10^{3} /$ year). There were no significant differences between years (Table 1). The average male ratios for newly diagnosed inguinal hernia cases and number of herniorrhaphies were $92.4 \%$ and $92 \%$, respectively (Table 1 ). In addition, the male ratios for both newly diagnosed inguinal hernia cases and number of herniorrhaphies increased significantly by age; however, there were no significant differences between years (Table 2). About $59.5 \%$ of the newly-diagnosed inguinal hernia cases received herniorrhaphy. Numbers of newly diagnosed inguinal hernia cases, number of herniorrhaphies, and incidence rates were highest in patients $>70$ years, followed

Table I Newly diagnosed inguinal hernia cases, sex difference, and numbers of herniorrhaphies performed from 200I to 2008

\begin{tabular}{|c|c|c|c|c|c|c|}
\hline Year & NDC & MR & IR & $H x(n)$ & MR & IR \\
\hline 2001 & I,592 & 92.4 & 1.71 & 800 & 92.0 & 0.86 \\
\hline 2002 & I,597 & 92.0 & 1.71 & 889 & 91.9 & 0.95 \\
\hline 2003 & I,458 & 92.0 & 1.53 & 821 & 92.0 & 0.86 \\
\hline 2004 & I,59| & 92.8 & 1.66 & 983 & 91.6 & 1.03 \\
\hline 2005 & 1,510 & 92.8 & $|.5|$ & 926 & 92.4 & 0.93 \\
\hline 2006 & I,426 & 92.6 & $\mathrm{I} .43$ & 945 & 92.1 & 0.95 \\
\hline 2007 & I,326 & 92.0 & 1.34 & 846 & 92.8 & 0.85 \\
\hline 2008 & I,228 & 92.0 & 1.24 & 765 & 91.5 & 0.77 \\
\hline Mean & 1,466 & 92.4 & 1.52 & 871.9 & 92.0 & 0.86 \\
\hline
\end{tabular}

Abbreviations: NDC, newly diagnosed inguinal hernia cases; MR (\%), male ratio (number of male patients/total patients $\times 100 \%$ ); IR, incidence rate (per I03/year); $\mathrm{Hx}$, herniorrhaphy. 
Table 2 Newly diagnosed inguinal hernia cases, sex difference, and numbers of herniorrhaphies performed by age group from 2001 to 2008

\begin{tabular}{llll|ll}
\hline Age (years) & NDC & MR & \multicolumn{1}{l}{ IR } & Hx (n) & MR \\
\hline$<10$ & 2,070 & 89.9 & $55.7 \mathrm{I}$ & $\mathrm{I}, 236$ & 89.5 \\
$10-19$ & 340 & 90.6 & 5.21 & 202 & 90.5 \\
$20-29$ & 815 & 91.6 & 3.12 & 493 & 91.4 \\
$30-39$ & 765 & 91.7 & 3.86 & 465 & 91.6 \\
$40-49$ & 1,240 & 92.9 & 7.72 & 748 & 92.5 \\
$50-59$ & 1,670 & 93.6 & $15.6 \mathrm{I}$ & $\mathrm{I}, 000$ & 93.0 \\
$60-69$ & 1,895 & 93.7 & 20.12 & $\mathrm{I}, 136$ & 93.5 \\
$70+$ & 2,933 & 95.2 & 68.91 & 1,696 & 94.0 \\
\hline
\end{tabular}

Abbreviations: NDC, newly diagnosed inguinal hernia cases; MR (\%), male ratio (number of male patients/total patients $\times 100 \%$ ); IR, incidence rate (per 103/year); $\mathrm{Hx}$, herniorrhaphy.

by those that aged $60-69,50-59$, and $40-49$ years (Table 2). The numbers of newly diagnosed inguinal hernia cases and number of outpatient visits for inguinal hernia were highest during summer, followed by spring, autumn, and winter, with significant difference. In addition, the number of herniorrhaphies was highest during summer, followed by spring, winter, and autumn, with significant difference (Table 3). Numbers of newly diagnosed inguinal hernia cases and outpatient visits for inguinal hernia were highest in the north of Taiwan and lowest in eastern Taiwan (Table 4), and this difference was significant after adjustment for population distribution in the different areas (47\% in the north, $25 \%$ in the center, $24 \%$ in the south, and $4 \%$ in the east). Lastly, only 2.49 per $10(5)$ per year of male patients who received herniorrhaphy developed ipsilateral varicocele; a higher incidence was found in patients aged 10-19 years and a lower incidence in those older than 50 years (Table 5 ). The average duration of developing ipsilateral varicocele after herniorrhaphy was 6.5 (range 3.2-10.4) years.

\section{Discussion}

More than $90 \%$ of inguinal hernias occur in men, and especially in elderly men, and surgical intervention is recommended given the risk of bowel obstruction or strangulation. ${ }^{18,19}$ In the current study, we found that the male ratios for both newly diagnosed inguinal hernia cases and number of herniorrhaphies increased significantly according to age, and that $92.4 \%$ of inguinal hernias occurred in men and especially in those older than 70 years. A possible explanation for this is that aging causes the connective tissue to become weak, thereby decreasing abdominal wall strength, and because older men have a higher incidence of prostatism. ${ }^{2}$ Further, $91.9 \%$ of herniorrhaphies were performed in men. This is the first study to report that the numbers of newly diagnosed inguinal hernia cases and outpatient visits for inguinal hernia are highest in summer. A possible explanation is that the higher temperature in summer may induce a higher rate of inguinal and scrotal discomfort, thereby increasing the number of outpatient visits for inguinal hernia. Another possible reason might be that the patient and family have more free time during the summer vacation, but the etiology warrants further evaluation. ${ }^{20}$

The incidence of inguinal hernia in infertile Israeli men has been reported to be $6.65 \%{ }^{21}$ Patients with a varicocele may complain of a dull throbbing scrotal pain or pulling or dragging sensation that is worsened with straining and exercise $; 2$ however, this scrotal pain may be due to inguinal hernia. Our previous study showed two patients $(8 \%)$ with asymptomatic varicocele that became painful varicocele after herniorrhaphy during follow-up, and both of the patients had an indirect inguinal hernia and a smaller body mass index. A possible explanation may be adhesion after dissection, obstructing venous return in the spermatic cord, and a lower body mass index may have disadvantages in relieving the nutcracker phenomenon. ${ }^{23}$ In the current study, we found a smaller risk of developing ipsilateral varicocele after herniorrhaphy, but clinicians should pay attention to patients in the age group of 10-19 years. Some issues might have an impact on the age factor. First, use of a different surgical technique for herniorrhaphy was noted between younger and older patients, and many younger patients received high ligation only without posterior wall repair. Second, pubertal boys and some adolescents still had testicular growth after hernior-

Table 3 Newly diagnosed inguinal hernia cases, numbers of outpatient visits, and numbers of herniorrhaphies performed in different seasons between $200 \mathrm{I}$ and 2008

\begin{tabular}{|c|c|c|c|c|c|c|c|c|}
\hline \multirow[t]{3}{*}{ Parameters } & \multicolumn{8}{|c|}{ Season } \\
\hline & \multicolumn{2}{|c|}{ Spring } & \multicolumn{2}{|c|}{ Summer } & \multicolumn{2}{|c|}{ Autumn } & \multicolumn{2}{|c|}{ Winter } \\
\hline & $\bar{n}$ & $\%$ & $\bar{n}$ & $\%$ & $\bar{n}$ & $\%$ & $\bar{n}$ & $\%$ \\
\hline $\mathrm{NDC}^{\dagger}$ & 2,832 & 25.46 & 2,976 & 26.75 & 2,809 & 25.25 & 2,507 & 22.54 \\
\hline $\mathrm{OV}^{*}$ & 9,853 & 25.13 & 10,798 & 27.54 & 9,968 & 25.42 & 8,596 & 21.92 \\
\hline$H x^{\#}$ & $\mathrm{I}, 875$ & 25.33 & 2,019 & 27.28 & $|, 88|$ & $25.4 \mathrm{I}$ & I,627 & 21.98 \\
\hline
\end{tabular}

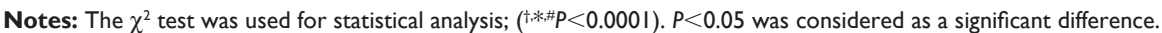

Abbreviations: NDC, newly-diagnosed inguinal hernia cases; OV, outpatient visits; $\mathrm{Hx}$, herniorrhaphies. 
Table 4 Newly diagnosed inguinal hernia cases and numbers of outpatient visits by area of Taiwan from 200 I to 2008

\begin{tabular}{|c|c|c|c|c|c|c|c|c|}
\hline \multirow[t]{3}{*}{ Parameters } & \multicolumn{8}{|c|}{ Areas } \\
\hline & \multicolumn{2}{|c|}{ Northern } & \multicolumn{2}{|c|}{ Central } & \multicolumn{2}{|c|}{ Southern } & \multicolumn{2}{|c|}{ Eastern } \\
\hline & $\mathbf{n}$ & $\%$ & $\mathbf{n}$ & $\%$ & $\mathbf{N}$ & $\%$ & $n$ & $\%$ \\
\hline $\mathrm{NDC}^{\dagger}$ & 5,261 & 47.29 & 3,092 & 27.80 & 2,549 & 22.91 & 222 & 2.00 \\
\hline OV* & 6,250 & 47.40 & 3,658 & 27.74 & 3,013 & 22.85 & 266 & 2.02 \\
\hline
\end{tabular}

Notes: The $\chi^{2}$ test was used for statistical analysis $\left({ }^{+} P<0.000 I ; * P<0.000 I\right), P<0.05$ was considered as a significant difference.

Abbreviations: NDC, newly diagnosed inguinal hernia cases; OV, outpatient visits.

rhaphy. Third, younger subjects were more likely to undertake strenuous physical activity compared with older subjects. In addition, the incidence of varicocele without herniorrhaphy is about 0.61 (per $10^{3} /$ year), ${ }^{20}$ which is significantly higher than the incidence of post-herniorrhaphy varicocele $(2.49$ [per $10^{5} /$ year]) according to the National Health Insurance Research Database. However, the association between herniorrhaphy and development of post-herniorrhaphy varicocele is still unclear, and compared with the high prevalence of varicocele in the general population, the chance of postherniorrhaphy varicocele is trivial and clinical insignificant. Further prospective studies are warranted to elucidate the exact mechanism with a longer follow-up period and more cases because the etiology of varicocele is still unclear. Additionally, the presence of varicocele does not necessarily lead to infertility.

Numbers of newly diagnosed inguinal hernia cases and outpatient visits for inguinal hernia were highest in northern Taiwan, even after adjustment for population distribution. This may be because more people live in northern Taiwan and more medical and educational resources are available there compared with other areas of Taiwan. Therefore, the government should pay attention to balancing the distribution of resources in different regions of Taiwan. Further, the accessibility or availability of health care resources in the different areas may result in delayed surgical intervention for inguinal hernia, resulting in more complications or an

Table 5 Development of ipsilateral varicocele after herniorrhaphy in different years and different age groups

\begin{tabular}{lll|lll}
\hline Year & NDCV & \multicolumn{1}{l}{ IR } & Age & NDCV & IR \\
\hline 2001 & 17 & 1.83 & $<10$ & 15 & 1.54 \\
2002 & 25 & 2.67 & $10-19$ & 40 & 4.45 \\
2003 & 22 & 2.31 & $20-29$ & 31 & 3.47 \\
2004 & 31 & 3.24 & $30-39$ & 26 & 2.64 \\
2005 & 34 & 3.40 & $40-49$ & 35 & 3.51 \\
2006 & 17 & 1.71 & $50-59$ & 18 & 1.82 \\
2007 & 23 & 2.32 & $60-69$ & 15 & 1.56 \\
2008 & 24 & 2.43 & $70+$ & 13 & 1.44 \\
\hline
\end{tabular}

Abbreviations: NDCV, newly diagnosed cases of varicocele; IR, incidence rate (per 105/year). incarcerated hernia. However, Kingsnorth et al showed that the outcome of herniorrhaphy in Ghana is comparable with that reported in the literature, but found a higher incidence of severe cases. ${ }^{24}$ In our study, we did not compare such differences in the four geographic areas, and more evaluations are needed.

There are many methodological advantages to this study. First, it is a nationwide investigation and highly representative with little selection bias. Second, it was easy to obtain longitudinal records for a large number of patients from different geographical areas for age-stratified analysis without compromising the necessary sample size. ${ }^{25}$ Third, diagnosis and surgery were based on ICD codes, which reduced the possibility of confounding factors. ${ }^{26}$ Nonetheless, the study also has several limitations. First, potential disease and operation misclassification bias may exist. ${ }^{27}$ Second, there was no access to data on body mass index, patterns of exercise, occupation, smoking, alcohol consumption, and socioeconomic status in this study, which might confound the results. ${ }^{25}$ Third, the study was hindered by aggregation and lag time bias. Therefore, we cannot show which patients received herniorrhaphy or their characteristics. Additionally, some cases would have received herniorrhaphy more than once, including the other side and redo surgery, which might impact the results.

In conclusion, numbers of newly diagnosed inguinal hernia cases and outpatient visits for inguinal hernia were highest during summer and lowest in eastern Taiwan. The male ratios for both newly diagnosed inguinal hernia cases and number of herniorrhaphies increased significantly with age. In addition, the risk of developing ipsilateral varicocele after herniorrhaphy in male patients was low, but was higher in patients aged 10-19 years.

\section{Acknowledgment}

This study was supported by a grant (99002-62-006) from Taipei City Hospital, Taiwan.

\section{Disclosure}

The authors report no conflicts of interest in this work. 


\section{References}

1. Holzheimer RG, Schreiber A. Inguinal hernia and concomitant varicocele mimicking mesh complication. Eur J Med Res. 2003;8:254-256.

2. Ruhl CE, Everhart JE. Risk factors for inguinal hernia among adults in the population. Am J Epidemiol. 2007;165:1154-1161.

3. Kingsnorth A, LeBlanc K. Hernias: inguinal and incisional. Lancet. 2003;362:1561-1571.

4. Hendry PO, Paterson-Brown S, de Beaux A. Work-related aspects of inguinal hernia: a literature review. Surgeon. 2008;6:361-365.

5. Abrahamson J. Etiology and pathophysiology of primary and recurrent groin hernia formation. Surg Clin North Am. 1998;78:953-972.

6. Liem MS, van der GraafY, Beemer FA, van Vroonhoven TJ. Increased risk for inguinal hernia in patients with Ehlers-Danlos syndrome. Surgery. 1997;122:114-115.

7. Klinge U, Binnebosel M, Mertens PR. Are collagens the culprits in the development of incisional and inguinal hernia disease? Hernia. 2006;10:472-477.

8. Pans A, Albert A, Lapiere CM, Nusgens B. Biochemical study of collagen in adult groin hernias. J Surg Res. 2001;95:107-113.

9. Nikolov S, Beltschev B. [Several ultrastructural peculiarities of the fascia transversalis in direct inguinal hernias of senile men]. Anat Anz 1990;170:265-272. German.

10. Ozdemir S, Ozis ES, Gulpinar K, et al. The value of copper and zinc levels in hernia formation. Eur J Clin Invest. 2011;41:285-290.

11. Ribarski I, Lehavi O, Yogev L, et al. USP26 gene variations infertile and infertile men. Hum Reprod. 2009;24:477-484.

12. Witt MA, Lipshultz LI. Varicocele: a progressive or static lesion? Urology. 1993;42:541-543.

13. Gorelick JI, Goldstein M. Loss of fertility in men with varicocele. Fertil Steril. 1993;59:613-616.

14. Lu JFR, Hsiao WC. Does universal health insurance make health care unaffordable? Lesions from Taiwan. Health Aff. 2003;22:77-88.

15. Chiang TL. Taiwan 1995 healthcare reform. Health Policy. 1997;39: 225-239.

16. Directorate-General Budget, Accounting and Statistics. National Statistics of Regional Standard Classification Data. Taipei, Taiwan: Accounting and Statistics; 1993.
17. Chen YK, Liu HC, Chen CS, Yeh SD. Seasonal variations in urinary calculi attacks and their association with climate: a population-based study. J Urol. 2008;71:581-585.

18. Abramson JH, Gofin J, Hopp C, Markler A, Epstein LM. The epidemiology of inguinal hernia. A survey in western Jerusalem. J Epidemiol Community Health. 1978;32:59-67.

19. Alvarez JA, Baldonedo RF, Bear IG, Solis JA, Alvarez P, Jorge JI. Incarcerated groin hernias in adults: presentation and outcome. Hernia. 2004;8:121-126.

20. Liu WL, Chen YA, Lai YW, Hsueh TY, Chen SS, Chiu AW. Nationwide survey to evaluate the characteristics of medical utilization in patients with varicocele in Taiwan. Urological Science. 2013. doi: 10.1016/J. urols.2013.08.00.

21. Yavetz H, Harash B, Yogev L, Homonnal ZT, Paz G. Fertility of men following inguinal hernia repair. Andrologia. 1991;23:443-446.

22. Yaman O, Ozdiler E, Anafarta K, Gogus O. Effects of microsurgical subinguinal varicocele ligation to treat pain. Urology. 2000;55:107-108.

23. Chen SS, Huang WJ. The experience of management of varicocele during ipsilateral inguinal herniorrhaphy, a prospective study. J Chin Med Assoc. 2010;73:248-251.

24. Kingsnorth AN, Clarke MG, Shillcutt SD. Public health and policy issues of hernia surgery in Africa. World J Surg. 2009;33:1188-1193.

25. Jollis JG, Ancukiewicz M, Delong ER, Pryor DB, Muhlbaier LH, Mark DB. Discordance of database designed for claims payment versus clinical information systems: implications for outcomes research. Ann Intern Med 1993;119:844-850.

26. Chen HF, Chen P, Li CY. Risk of malignant neoplasms of liver and biliary tract in diabetic patients with different age and sex stratifications. Hepatology. 2010;52:155-163.

27. Lin CC, Lai MS, Syu CY, Chang SC, Tseng FY. Accuracy of diabetes diagnosis in health insurance claims data in Taiwan. J Formos Med Assoc. 2005;104:157-163.
Patient Preference and Adherence

\section{Publish your work in this journal}

Patient Preference and Adherence is an international, peer-reviewed, open access journal focusing on the growing importance of patient preference and adherence throughout the therapeutic continuum. Patient satisfaction, acceptability, quality of life, compliance, persistence and their role in developing new therapeutic modalities and compounds to

\section{Dovepress}

optimize clinical outcomes for existing disease states are major areas of interest. This journal has been accepted for indexing on PubMed Central. The manuscript management system is completely online and includes a very quick and fair peer-review system. Visit http://www.dovepress.com/ testimonials.php to read real quotes from published authors. 